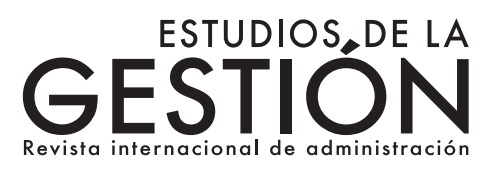

\title{
Ecosistema empresarial
}

\author{
Melquicedec Lozano \\ Universidad Icesi (Cali, Colombia) \\ melqui@icesi.edu.co
}

Fecha de presentación: 9 de agosto de 2015 • Fecha de aceptación: 23 de noviembre de 2016 Artículo de investigación 


\section{Resumen}

Los ecosistemas empresariales han tomado fuerza durante los últimos años debido a las fortalezas que tienen para favorecer el desarrollo del espíritu emprendedor y la creación de nuevas empresas; sin embargo, la literatura sobre ecosistemas empresariales todavía es escasa. Esta investigación parte del diseño de un ecosistema empresarial para determinar los componentes que lo deben conformar, cómo deben estar relacionados para interactuar organizadamente y cuáles han de ser las acciones a ejecutar.

Palabras clave: ecosistema empresarial, espíritu emprendedor.

JEL: L26 Emprendedores; I23 Centros de enseñanza superior y de investigación; M14 Cultura corporativa.

\section{Abstract}

Business ecosystems have become stronger in recent years due to the strengths they have in favor of the development of the entrepreneurial spirit and the creation of new companies; however, the literature on business ecosystems is still scarce. This research starts from the design of an enterprise ecosystem to determine the components that should conform it, how they should be related to interact in an organized manner and what the actions to be executed should be.

Keywords: business ecosystem, entrepreneurial spirit.

JEL: L26 Entrepreneurs; I23 Higher education and research centers; M14 Corporate Culture.

\section{Resumo}

A linha de trabalho sobre ecossistemas empresariais ganhou uma forte importância nos últimos anos devido à capacidade que estes possuem de favorecer o desenvolvimento do espírito empreendedor e a criação de novas empresas. No entanto, a literatura sobre ecossistemas empresariais é escassa, o que suscitou o interesse por aprofundar os conceitos referentes a tal tema. Assim, esta pesquisa parte do design de um ecossistema empresarial de forma a determinar quais são os componentes que devem formá-lo, como estes devem estar relacionados para interagir organizadamente e quais são as ações a serem executadas. Este trabalho pretende ajudar a esclarecer tal assunto.

Palavras-chave: Ecossistema empresarial, espírito empreendedor, recursos, novas empresas, empreendedor.

JEL: L26 Empreendedorismo; I23 Centros de ensino superior e de pesquisa; M14 Cultura corporativa. 


\section{Introducción}

1 fomento del espíritu empresarial como una estrategia de desarrollo económico (Ortmans 2015) ha llevado al concepto de "ecosistema empresarial". El espíritu empresarial emergido como una política prioritaria es parte de la respuesta a la decepción con las políticas industriales que poco resultado han propiciado en lo que se refiere a la generación de empleo, y a la debilidad de un desacertado énfasis en condiciones macroeconómicas particulares que por muchos años han permitido el funcionamiento de organismos sin la interacción suficiente para complementarse y operar como un sistema organizado y eficiente, deteriorando así la calidad de los servicios orientados hacia la comunidad. En atención a esto se requiere, por consiguiente, dar mayor relevancia a lo que significa ecosistema empresarial.

La creación de trabajo no ha sido el objetivo principal del fomento de un ecosistema empresarial (Isenberg 2013). Esto en realidad depende de quién es el stakeholder. Para el gobierno, el objetivo principal puede ser la creación de trabajo. Para los banqueros el objetivo puede ser la rentabilidad sobre los préstamos concedidos. Para las universidades probablemente la creación de conocimiento y la difusión que a partir de un ecosistema universitario se pueda derivar. Para los emprendedores la creación de riqueza y la autorrealización. Para las instituciones puede ser la innovación y el desarrollo de sus sistemas de operación. De ahí que los ecosistemas empresariales guarden un perfil que se ajusta en buena medida a los intereses de sus gestores.

A lo anterior se agrega que no hay una evidencia sistemática acerca de que los espacios de coworking contribuyan significativamente a la aparición de nuevos emprendimientos. Aunque esto evidentemente no hace mal, se perciben muchos casos de emprendimientos exitosos que no han usado espacios de coworking, como las incubadoras, pues algunos emprendedores no acceden por diversas razones como la distancia, el cumplimiento de normas 
y las formas contractuales con estas unidades de apoyo. Otros no los utilizan argumentando que disminuyen su autonomía y creatividad. Otros, sin embargo, argumentan que el acceso a redes provisto por los espacios de coworking les da acceso a información e ideas que les favorecen. Lo cierto es que los mecanismos de soporte creados intencionalmente son solo un elemento en el paquete de componentes de un ecosistema empresarial, por lo cual, en muchos emprendimientos es un elemento ausente.

Por otro lado, realza Isenberg (2013) que la educación formal en entrepreneurship no necesariamente lleva a emprendimientos exitosos. Hay evidencia de que escenarios de acceso empresarial como Israel, Ruta 128, Silicon Valley, Austin, Iceland, Skulkovo (Rusia), Kenza Techno City (Kenia), Porto Digital (Brasil), New Songdo (Corea del Sur), Mediápolis (Singapur), el Barrio Tecnológico de Espoo (Finladia), Milla Digital (España), el Cluster de Santa Fe (México), Tech City (Inglaterra), Technopark (Suiza), Technology Cluster (Hong Kong) y Cyberjava ( Malasia), tuvieron espíritu empresarial mucho antes de que en estos puntos hubiera cursos sobre entrepreneurship. Se comenta en algunos casos que los medios típicos que se necesitan para fomentar un ecosistema empresarial (aceleradoras, préstamos, etc.) no ayudan profundamente en sí mismos, lo más importante es que los actores en el ecosistema estén alineados y tengan objetivos claros. En contraste, Isenberg (2013) también señala que con los emprendedores por sí mismos no habría ecosistema empresarial; los emprendedores son piezas esencialmente requeridas, pero no son lo único. Incluso, no necesariamente un ecosistema empresarial es manejado por un emprendedor. En un ecosistema empresarial también deben existir grandes empresas, lo que favorece porque son clientes importantes y canales de mercado para los emprendedores, no necesariamente competidores, y también porque en las empresas grandes se forjan ejecutivos talentosos que toman decisiones movilizando los mercados.

Para los emprendedores los tres grandes retos son acceder al talento, superar la burocracia excesiva y acceder al escaso capital para iniciar emprendimientos; lo que está más del lado del proceso genérico del entrepreneurship que de una deficiencia del ecosistema. Así, el proceso da lugar al sentimiento de que el capital de riesgo es escaso y difícil de conseguir, lo cual no siempre es así (Čalopa, Horvat y Lalić 2014). Quizá por lo anterior, el énfasis sobre los apoyos para crear empresa se ha puesto desde tiempo 
atrás en el tema financiero (Matiz y Fracica 2011), a pesar de los otros temas que han surgido en este siglo y han ido tomando un lugar relevante, como el modelo de negocio, la investigación de mercados, el análisis de las operaciones y la estructura inicial de la empresa que inicia. Bien se ha expuesto sobre la posibilidad de varios canales de apoyo financiero para el surgimiento de empresas, sin asegurar que estos recursos sean fáciles de conseguir (Čalopa, Horvat y Lalić 2014). En este abanico de posibilidades, Isenberg (2013) señala que aunque se habla de recursos como los inversores ángeles, un canal altamente relevante para el surgimiento de empresas a nivel mundial, lo cierto es que hasta el momento no ha sido así. Señala también que, la mayoría de inversiones de capital de riesgo se han hecho en California, Nueva York, Massachusetts e Israel, usando recursos que en su mayoría no han sido de inversores ángeles.

Atendiendo los señalamientos expuestos, para que un ecosistema empresarial funcione de manera apropiada y útil se requiere de varios factores que confluyan y actúen disciplinadamente como un todo. Hay factores indispensables en el ecosistema que por sí solos no alcanzarían la eficiencia deseable.

\section{Surgimiento y relevancia}

El concepto de ecosistema ha ido evolucionando paulatinamente desde que la teoría de ecosistemas empresariales se originó en Moore (1993), quien señaló que el ecosistema de negocios era un espacio de interconexión y dependencia entre agentes económicos que debía funcionar de manera saludable como condición indispensable para que las organizaciones tuviesen éxito y continuidad. Este concepto guarda relación con lo expresado en la introducción, en cuanto que un escenario para favorecer la aparición de empresas y hacer que se desarrollen se enmarca en un contexto en el que concurren emprendedores, universidades, conocimiento, redes, capitales de riesgo, infraestructura, laboratorios, asesores, política pública, acuerdos privados, industrias y fuerza laboral. De ahí que el término más difundido hoy sea el de ecosistema empresarial, también conocido como ecosistema de emprendimiento. Este es un término usado para referir un paquete de condiciones y medidas políticas que se orientan a dar soporte a las necesidades que tienen 
las nuevas empresas durante su ciclo de vida inicial, condiciones y políticas que se basan con frecuencia en la concepción estrecha de cómo el espíritu empresarial contribuye al crecimiento económico (Moore 1993). En respuesta a esta supuesta falencia surgen propuestas como el sistema nacional de entrepreneurship (Acs, Szerb y Autio 2015), el cual se define como la dinámica de interacción, integrada institucionalmente por individuos, entre actitudes empresariales, capacidades y aspiraciones, que lleva a la distribución de recursos por medio de la creación y operación de nuevos emprendimientos.

Willer y Neely (2013) dicen que un ecosistema de negocios se refiere a la red de una determinada empresa, sus proveedores, sus empresas complementarias y sus clientes, por lo que algunas características claves de los ecosistemas empresariales son las interconexiones de las empresas y los procesos de competición y cooperación. Sin embargo, existen pocas herramientas para el análisis de estrategias de las empresas en ecosistemas, aunque el mapeo de un ecosistema ha sido usado como una herramienta visual para entender las relaciones e interdependencias entre empresas que tienen presencia dentro de él. Estos autores se enfocan en tratar de entender cómo los nuevos modelos de negocios son diseñados en el contexto de todo un ecosistema que no está todavía maduro y cómo la perspectiva del ecosistema puede guiar el diseño del modelo de negocio. También se proponen desarrollar una metodología para evaluar y comparar alternativas de diseños de modelos de negocios en un ecosistema emergente. Este propósito se acoge también en las disertaciones que exponen Zott y Amit (2010).

Debe reconocerse que Willer y Neely (2013) resaltan el modelo de economía empresarial, tratado previamente por otros autores (Audretsch y Thurik 2004), que ha reemplazado al modelo de la economía dirigida. Son dos modelos que contrastan en los diferentes roles que desempeña el entrepreneurship. El modelo de la economía dirigida gira, por un lado, en torno a enlaces entre estabilidad, especialización, homogeneidad, escala, certeza y predicción, y, por otro, en torno al crecimiento de la economía. En cambio, el modelo de la economía empresarial se orienta a los enlaces entre flexibilidad, turbulencia, diversidad, novedad, innovación, relaciones y agrupaciones, aunque también al crecimiento económico. En este modelo el conocimiento es el valor dominante de la producción, Situación que tiene una connotación muy importante, pues la ventaja comparativa en la economía del 
conocimiento depende en buena medida de la actividad innovadora (Nambisan y Baron 2013; Autio y otros 2014). Por esta razón, este modelo tiene fuertes fundamentos en el cambio. Así, debe hacerse, como es tendiente en la actualidad, una distinción entre innovaciones incrementales e innovaciones radicales. Las incrementales son aquellas compatibles con las competencias fuertes y la trayectoria tecnológica de la empresa (Teece y otros 1994); mientras que las innovaciones radicales son las que van más allá de los límites de las competencias fuertes y la trayectoria tecnológica de la empresa. En el modelo de la economía empresarial, la capacidad de romper paradigmas es impulsada por la capacidad de los agentes económicos para iniciar nuevas empresas. En este modelo, la ventaja comparativa de los altos costos de posicionamiento demanda actividades innovadoras más tempranas en el ciclo de vida del producto, lo que plantea una naturaleza más radical que incremental. Estas consideraciones llevan a que los sistemas empresariales cuenten con condiciones que favorezcan el surgimiento de innovaciones incrementales y radicales, pero en ningún caso deberían escatimar esfuerzos para incentivar permanentemente estas últimas.

Los dos tipos de innovación deben ser favorecidos por la legislación. Hay que recordar, como enfatiza Mattews (2002), que las condiciones del país tienen que ver con un marco legal que define aspectos como: el límite de precios, los intereses permitidos sobre préstamos del mercado financiero, los impuestos de diferente índole, los asuntos políticos de crecimiento y seguridad y las disposiciones que enmarcan el manejo laboral de las empresas. De la mano con estas condiciones también se considera el concepto de riesgo país, que se acoge para medir el riesgo de un estado en su proceso de consecución de divisas y resolver sus compromisos de moneda extranjera. De lo anterior se desprende que la competencia y la regulación son elementos claves en un ecosistema empresarial, por lo cual, los entes que supervisan y controlan la dinámica empresarial cada día se ven más presionados a prepararse a más alto nivel para asumir las funciones con objetividad y tomar decisiones que verdaderamente favorezcan los buenos resultados empresariales en su conjunto.

Es bien conocido que el éxito del fenómeno de Silicon Valley ha llevado a otros países a esforzarse por crear sus propias configuraciones productivas y legales. La mayoría de estos conglomerados incluye una o más universida- 
des líderes, centros de investigación, capital de riesgo, mano de obra calificada, programas para atraer la inversión, un fuerte mercadeo internacional y una exigente interacción con los organismos reguladores.

El caso típico y difundido del Silicon Valley (Leslie 2000) ha estimulado la configuración de zonas ya mencionadas anteriormente, y otras como Silicon Green, entre Edinburgo y Glasgow en Escocia; Silicon Bog en Irlanda; Silicon Wadi en Tel Aviv; Bangalore en la India y Silicon Island en Taiwán. En adición, Auletta y Rivera (2011) mencionan algunas experiencias que han ido fortaleciendo paulatinamente el marco empresarial en América Latina tales como: Buenos Aires Emprende (Argentina), Emprende Claro (Chile) y Ley 1014 (Colombia), México Emprende (México), Emprendedores en red (Uruguay) y Endeavor (en varias regiones del mundo). En estos espacios, la innovación y la comunicación son factores claves. Se parte de la definición de cluster como un sistema de empresas e instituciones interconectadas cuyo valor como un todo es mayor que la suma de sus partes (Porter 1998). Sin embargo, no son solo cluster (Cantwell y Glac 2004), se ha ido más allá, al requirir un ecosistema empresarial que los soporte.

\section{Características y componentes}

Este apartado recoge los puntos de vista de reconocidos autores que se han involucrado directamente con las características y componentes de un ecosistema empresarial. Sus disertaciones se han aprovechado para analizar los aspectos más cercanos al objetivo de este trabajo. Estas posiciones conllevan contribuciones que sirven de fundamento al siguiente apartado (una mirada desde los recursos).

En sus enunciados sobre las características del ecosistema empresarial, Hielema (2013) señala algunas que son: a) una red interconectada responsable de los emprendedores, b) todos sus elementos son necesarios para una economía próspera, c) no hay un elemento que apoye la iniciativa empresarial por su única cuenta, y d) un conjunto de recursos que tienen que trabajar juntos. De igual manera expone que un ecosistema básico podría tener los siguientes componentes: 
1. Abogados, que proporcionan la guía y el apoyo legal a las empresas e industrias.

2. Educadores empresariales, que proporcionan formación, grados y certificados.

3. Fuentes de financiación, que brindan financiamiento y capital para los empresarios.

4. Profesionales expertos, que ofrecen servicios de apoyo profesional.

5. Reconocimientos, premios y distinciones para exaltar el éxito empresarial.

6. Proveedores, que proporcionan orientación especializada y recursos de operación para los empresarios.

7. Fuentes de innovación, que soportan la propiedad intelectual y de comercialización.

8. Talento, empleados y trabajadores, provistos por universidades, centros de formación y organizaciones.

En este ecosistema básico los emprendedores están en el centro. Dependiendo de las comunidades puede haber otros componentes que puedan ser incluidos en el ecosistema, como en el caso de los ecosistemas empresariales universitarios (Carvalho, Costa y Dominguinhos 2010; Graham 2014; Duricova y otros 2014; Diaconu y Dutu 2015) que involucrarían componentes como los investigadores al interior de la universidad, o los medios experimentales materializados en máquinas o equipos específicos, o el conocimiento especializado que se transmitiría en el caso de crear empresas spin-off. Algunos ámbitos de nivel superior están prestos a dar cabida a lo expuesto por Hielema (2013); en particular, Carvalho, Costa y Dominguinhos (2010) resaltan que las instituciones de educación superior tienen un rol importante en el mejoramiento del entrepreneurship, pues hacen parte de un ecosistema empresarial, junto con las empresas y el gobierno. Para confirmarlo muestran el caso del ecosistema empresarial desarrollado en el Instituto Politécnico de Setúbal (SPI), en Portugal, que considera tres dimensiones: cursos curriculares de entrepreneurship, programas extracurriculares del entrepreneurship y soporte infraestructural. Los autores intentan proveer una visión integrada de estas tres dimensiones y esbozan los vínculos con la comunidad y las redes existentes que pretenden estimular el capital social y facilitar los procesos empresariales. El SPI también fomenta el aprendizaje e interacción de los emprendedores mediante eventos y actividades tales como 
start-up weekends, tech meeet-ups, venture pitches, young entrepreneurs organization y otros. Desde otro ángulo, instituciones como las cámaras de comercio, cuyo propósito no es la educación superior, cumplen un papel complementario en mayor o menor grado en casi todos los países. Un caso es la Cámara de Comercio Regional de Orlando, la cual, justamente, estuvo dirigida por Hielema (2013) hasta hace algunos años, y proporciona apoyo en lo que se denomina "ecosistema de exportación internacional", con una activa web-site, unas herramientas de navegación, así como una conexión y coordinación tecnológica del ecosistema empresarial que favorezca a los emprendedores. Otro papel desempeñan las incubadoras de empresas en muchos países, como Bélgica con Le Magellan o Colombia con Incubar, solo por mencionar estos dos, que engranan con apoyos a los emprendedores, bien sea con espacios, uso de servicios con costo compartido o asesoría financiero-comercial.

Dando lugar a otro componente importante, Clarysse y otros (2014) realzan el conocimiento como parte fundamental de los ecosistemas empresariales y acogen el concepto de ecosistema de conocimiento. Señalan que crear un denso ecosistema de conocimiento mantiene la garantía de estimular un alto grado de innovación en un área determinada. En torno a esto hacen mención a dos características y un mecanismo que son centrales en el desarrollo de sistemas de conocimiento: a) una diversidad de formas de organización, b) la presencia de un inquilino ancla, y c) el mecanismo de transposición entre ámbitos. Las organizaciones pueden ser universidades, centros de investigación, organizaciones emprendedoras, empresas de capital de riesgo, empresas de servicios, empresas de producción, u otro tipo de organizaciones. Algunas de estas instituciones podrían operar también como inquilinos ancla, siempre que no compitan con las otras organizaciones que habitan en la comunidad. Es común, por ejemplo, que alguna universidad local cumpla con el rol de inquilino ancla en los procesos de generación de conocimiento. De manera complementaria, existe alta fortaleza cuando las ideas y modelos son transportados de una red de formas organizacionales a otra. Estos autores resaltan que un ecosistema de conocimiento tiene su foco en la generación de conocimiento, mientras que un ecosistema empresarial lo tiene en la creación de valor para el cliente; y también que la conectividad de los actores en un ecosistema de conocimiento se da en un cluster geográfico, mientras en un ecosistema empresarial se da en el valor de la red. Clarysse y otros (2014) se- 
ñalan que parece haber una desconexión entre el desarrollo de conocimiento y los ecosistemas empresariales, y recomiendan revisar las políticas de los procesos de creación de valor en ambas partes para lograr mejores conexiones y resultados conjuntos. Esta aseveración parece ser más entendida en los centros de investigación que operan desde las universidades o en los que operan de manera privada, razón por la cual tienen definidas algunas políticas de transferencia de conocimiento y de respaldo a la creación intelectual.

En este recorrido por las características y componentes de un ecosistema empresarial, Isenberg (2010) describe en sus enunciados el entorno en el cual el espíritu empresarial tiende a prosperar. Propone que los emprendedores son más exitosos cuando tienen acceso a recursos humanos, financieros y profesionales y cuando pueden actuar en un entorno en el que las políticas del gobierno fomentan y apoyan a los empresarios. Describe estos recursos y este tipo de entorno como el ecosistema empresarial y muestra nueve principios para la construcción de un ecosistema empresarial exitoso, los que deben integrarse holísticamente:

1. Dejar de emular a Silicon Valley.

2. Formar el ecosistema alrededor de condiciones locales.

3. Involucrar al sector privado.

4. Favorecer a los emprendedores de alto potencial.

5. Obtener una gran victoria.

6. Encarar de frente el cambio cultural y los retos.

7. Resaltar las raíces del arranque.

8. Ayudar a los emprendedores a crecer de forma orgánica.

9. Tener en cuenta reformas legales, regulaciones y escenarios burocráticos. Además de los anteriores nueve principios, Isenberg (2010) propuso un atractivo análisis de seis componentes de un ecosistema empresarial, cuyas definiciones se resumen así:

- Mercados: En este componente situó las redes de emprendedores (redes de personas en diáspora, corporaciones multinacionales) y los clientes iniciales (pruebas de concepto, clientes referidos, canales de distribución, entre otros).

- Política: En este componente se refirió a liderazgo (soporte indiscutible, legitimidad social, caminos para defenderse, estrategia de espíritu em- 
prendedor, crisis y retos) y gobierno (soporte institucional, soporte financiero, marco regulatorio de control e incentivos, centros e institutos de investigación, marco legal para contratos, derechos de propiedad, entre otras).

- Finanzas: Capital financiero (préstamos, inversores ángeles, fondos de capital de riesgo, mercados públicos de capital, deuda y capitales privados).

- Cultura: Historias de éxito (éxito visible, generación de riqueza para los fundadores y reputación internacional) y normas sociales (tolerancia al riesgo y a las fallas, creatividad e innovación con experimentación, estatus social de emprendedor, creación de riqueza, ambición e impulso).

- Soportes: Instituciones no gubernamentales (promoción del espíritu empresarial, concursos de plan de empresa, conferencias y asociaciones de emprendedores), profesiones de soporte (abogados, expertos en contabilidad, banqueros de inversión, asesores y técnicos expertos) e infraestructura (telecomunicaciones, transporte, logística, energía, servicios públicos, áreas, incubación, centros y clusters).

- Capital humano: Mano de obra (calificado, emprendedores y generaciones familiares adultas) e instituciones educativas (grados técnicos y profesionales, formación específica en entrepreneurship).

Estos seis componentes enunciados por Isenberg (2010) dan forma clara al tipo de componentes que deben considerarse en un ecosistema empresarial, pero no hace exhaustivo el concepto. En la contribución que hacen Mason y Brown (2014) definen el ecosistema empresarial como un conjunto de actores (existentes y potenciales), organizaciones (empresas, capitalistas de riesgo, bancos y ángeles inversores), instituciones (universidades, empresas públicas y centros especializados), y procesos (tasas de nacimiento, número de emprendedores y nivel de ambición empresarial), los cuales formal e informalmente se unen para concretar, mediar y regir el desempeño dentro del entorno empresarial local. Así mismo, el concepto de ecosistema empresarial es holístico e interactivo por naturaleza, por lo tanto, retoma conceptos como clusters, distritos industriales, sistemas de innovación y aprendizajes regionales. Se va evidenciando, por lo tanto, que lo que hace al ecosistema empresarial distinto de otras aproximaciones es que pone al empresario en el centro; de esta manera, lo que es analizado es la capacidad del entorno local 
para apoyar a los empresarios ambiciosos, en lugar de meramente la innovación o el desarrollo económico.

En complemento, Mazzarol (2014) retoma el concepto de Isenberg (2010) y dice que un ecosistema empresarial se refiere a la interacción que toma lugar entre actores individuales e institucionales para fomentar el espíritu empresarial, la innovación y el crecimiento de pequeñas y medianas empresas. Además, en su trabajo ajusta el modelo formulado por Isenberg (2010) y sugiere nueve componentes adicionales para el ecosistema empresarial: políticas de gobierno, marco regulatorio e infraestructura, fuentes de financiación, cultura, mentores, asesores y sistemas de soporte, y universidades como catalizadores, educación y formación, capital humano, y fuerza de trabajo y mercados locales y globales.

En realidad, al examinar en detalle, lo que hace Mazzarol (2014) es desagregar los seis componentes de Isenberg (2010) y enfatizar que quizá el componente más importante de un ecosistema empresarial corresponde a las políticas gubernamentales, aquellas directamente relacionadas con tasas, servicios financieros, telecomunicaciones, transporte, mercados de trabajo, inmigración, industrias de soporte, educación y formación, infraestructura y salud. En su disertación recuerda que los emprendimientos nuevos y las pequeñas empresas desempeñan un papel significativo en la creación de empleos, lo que explica el interés de los gobiernos en las políticas que favorecen a estas empresas y también el fomento del espíritu empresarial. En torno a esto, desde los enunciados de Mason y Brown (2014) se plantea que en la política que se establezca para los sistemas empresariales se debe dar atención a cuatro grupos principales que se ilustran en la figura 1.

De esta manera, Mazzarol (2014) se refiere en primera instancia a los actores empresariales, incluyendo a los emprendedores nacientes que están en un nivel pre-start o que ya están inmersos en actividades start-up de las nuevas creaciones de empresa. También están considerados aquellos que han adquirido empresas ya establecidas para revitalizarlas.

En segunda instancia hace referencia a los proveedores de recursos empresariales, incluidas las fuentes financieras, resaltando que la política debería hacer realidad los canales para acceder con facilidad a los fondos, particularmente orientados al crecimiento de las pequeñas y medianas empresas. $\mathrm{Su}$ percepción es que lo típico es hacerlo a través de capitales de riesgo, tales 
Figura 1

\section{Grupos centrales para la política de ecosistemas empresariales}

\section{Actores}

- Soporte y servicios de mentoría.

- Incubadores y espacios de coworking.

- Programas de networking y de aceleración.

\section{Proveedores de recursos}

- Financieros (bancos, capital de riesgo).

- Redes de inversores ángeles.

- Multifinanciación y préstamos de pares.

- Acceso al mercado de activos.

- Alianzas con universidades y centros de I y D.

\section{Política de sistema empresarial}

\section{Conectores}

- Asociaciones profesionales.

- Clubes de entrepreneurship.

- Centros de empresas.

- Servicios de búsqueda de inversores.

- Corredores de negocios.

\section{Orientación empresarial}

- Estatus social de autoempleo, pequeños negocios y emprendimientos.

- Redes de inversores ángeles.

- Modelo de roles.

- Educación en entrepreneurship.

- Tolerancia a las equivocaciones

e incorporación de la innovación.

Fuente: Mazzarol (2014).

Elaboración propia.

como las redes de ángeles inversores, los fondos de capital de riesgo y el mercado bancario, pero recalca que la política debe incentivar otras formas de financiación como el crowdfunding, por ejemplo. También considera que los emprendedores deben recibir formación para tener conocimiento y hacer una buena selección entre las opciones de financiación que estén a su alcance.

El otro grupo al cual se refiere Mazzarol (2014) es el de los conectores empresariales, tales como las asociaciones de profesionales, los grupos industriales, las cámaras de comercio e industria, los clubes de entrepreneurship, las comunidades start-up, las redes empresariales y los corredores de bolsa. La política de gobierno ha de estimular la existencia, expansión y buen funcionamiento de estos conectores.

Por último, Mazzarol (2014) alude a la formación empresarial, en clara referencia a la cultura de la región o país, una cultura propensa al riesgo y con voluntad para buscar el éxito, que examine modelos de roles de individuos y empresas, que proponga programas para incentivar el espíritu emprendedor y que los ciudadanos se esfuercen por acceder a ellos y sugiere que estos pro- 
gramas no sean solo de contenido sino que también entrenen a los emprendedores en habilidades que son requeridas en los negocios. La política también debe impulsar la inmigración para favorecer las inversiones y las alianzas. Adicionalmente, debe propiciarse una cultura de voluntad para generar nuevas ideas, innovar y acoger el cambio.

Para complementar sus planteamientos, Mazzarol (2014) le da acogida a los siete intangibles para el entrepreneurship regional formulados por Venkataraman (2004):

1. Puntos focales capaces de producir nuevas ideas. Debería de haber universidades, incubadoras, unidades, centros de investigación y desarrollo, que puedan servir para estimular nuevas innovaciones.

2. La necesidad de difundir modelos del rol. Emprendedores o empresas que deben servir como ejemplo de riesgo, de trabajo y de resultados.

3. La necesidad de foros informales de entrepreneurship. La actividad de entrepreneurship y el fomento de la innovación es más un proceso social que económico. Tiene que haber foros sin tanta formalidad que ayuden a facilitar los procesos de construcción de capital social.

4. La necesidad de ideas de creación de empresa enmarcadas en la región. Las tecnologías y modelos de negocio deberían surgir con relevancia para la región y poder crecer orgánicamente dentro del panorama industrial.

5. La necesidad de redes que protejan al emprendedor. El entrepreneurship implica la toma de riesgos y que el emprendimiento no prospere, por lo cual debería haber seguridad en la redes para que exista tolerancia al riesgo y a las fallas, así como leyes fiscales y de quiebras y un sistema de apoyo de bienestar social que no sancione a los emprendedores que fallan.

6. La necesidad de entradas a mercados grandes. Los emprendimientos que tienen potencial de crecimiento requerirán asegurar el acceso a mercados nacionales o internacionales.

7. La necesidad de liderazgo ejecutivo. La calidad del liderazgo es un elemento importante en el éxito de los nuevos emprendimientos, por lo cual son vitales los equipos de emprendedores con habilidades, unidad y determinación para hacer crecer sus negocios y volverlos exitosos.

Estos matices parecen llenar los vacíos que faltaban por cubrir en el recorrido de características y componentes examinados hasta aquí, proporcio- 
nando, de esta manera, los fundamentos necesarios para exponer, con una mirada desde los recursos, los componentes relevantes en un ecosistema empresarial y que se especificarán en el siguiente apartado.

Conviene finalizar este apartado haciendo mención a la importancia que empieza a tener la medición dentro de los ecosistemas empresariales. Capelleras y Kantis (2009) resaltan que la preocupación por el surgimiento y el rápido crecimiento de nuevas empresas es paralela al interés de estudiar y tener claridad sobre lo que deben ser, hacer y producir los ecosistemas para emprendedores (Valdés y Vidal 2012), pues de estos dependen, en buena medida, que nazcan empresas dinámicas que se desarrollen y que tengan perdurabilidad con operaciones y finanzas atractivas (Parker y Storey 2010). Esto ha conducido a que recientemente se estén midiendo las capacidades sistémicas del emprendimiento dinámico con el denominado ICSED-Prodem, que se basa en diez dimensiones clave que inciden sobre la cantidad y calidad de las nuevas empresas que se crean (Kantis, Federico e Ibarra 2014). Pero también la metodología Global Entrepreneurship and Development Index (GEDI) (Acs, Szerb y Autio 2015) captura las características centrales del sistema nacional de entrepreneurship que son: a) la contextualización de la información del nivel individual. Esto es, la noción de que las actividades del nivel individual son reguladas por el contexto; b) el uso de quince medidas contextuales de actitudes, habilidades y aspiraciones empresariales; es decir, la noción de que los procesos empresariales en el nivel país son complejos y multifacéticos; c) la aplicación del algoritmo Penalty for Bottleneck (sanciones para cuellos de botella) para capturar la noción de que los componentes del sistema coproducen unas salidas, y d) el reconocimiento de que el desempeño empresarial nacional puede ser frenado por factores de cuello de botella. Otro tipo de mediciones como las mencionadas por Monteanu, Iamandi y Tudor (2015) han surgido recientemente.

\section{Una mirada desde los recursos}

Por todo lo anteriormente expuesto, es indispensable reconocer que en torno al concepto de ecosistema empresarial se mueven y participan muchos elementos. Partiendo a inicios de la década de 1990 hasta ahora, se han he- 
cho algunos avances, aunque falta aún mucho por investigar y definir. Esto se corrobora con el reciente llamado que han hecho revistas científicas como Strategic Entrepreneurship Journal y Small Business Economics: An Entrepreneurship Journal, para que los investigadores postulen trabajos que ahonden en nuevos hallazgos sobre ecosistemas empresariales, específicamente. En la primera revista mencionada se espera publicar la respectiva versión en 2019, mientras en la otra se espera hacerlo antes. Seguramente, con estos y otros incentivos para que los investigadores profundicen científicamente en esta línea de trabajo, se generarán hallazgos valiosos que contribuirán a una conformación eficiente de los ecosistemas empresariales y al logro de mejores resultados. Sin embargo, es claro que los ecosistemas empresariales tienen algunos elementos comunes a todos, así como también han de tener elementos inherentes a su propia región, en términos de cultura, propósitos específicos, actores, recursos, interrelaciones, mercados foco, entre otros.

Como se pudo apreciar, Mazzarol (2014) ha destacado de manera particular la política de gobierno como el componente crucial entre los nueve que considera en sus exploraciones. De igual manera, otros observadores hacen planteamientos con otros puntos de vista. En atención a esto, en el presente trabajo se acoge una mirada al ecosistema empresarial desde los recursos como lo proponen García y García (2010); sin embargo, en contraste se consideran siete grupos de recursos en lugar de cinco, aportando para cada uno algunas consideraciones adicionales que no abordan García y García (2010); esta propuesta otorga más solidez al grupo de recursos físicos, denominándolos como recursos físicos e intangibles. Los siete grupos se muestran en la figura 2.

En el grupo de recursos humanos hay variables determinantes para crear: edad, motivaciones, nivel formativo, contexto familiar, valores sociales y cultura de la región. La formación es un factor vital, pues alimenta el capital humano de un ecosistema empresarial. Los valores sociales desempeñan un papel fundamental, pues tienen un efecto fuerte en el comportamiento (Duque, Cardona y Rendón 2013). Por ejemplo, en la comunidad se puede considerar que ser un empresario que cuida el medioambiente es deseable y que otorga un estatus cuando se tiene éxito. Sin embargo, en un ecosistema empresarial hay debilidades institucionales para la formación, como pueden ser los costos de acceso a la educación (Osorio y Pereira 2011), y debilidades 
Figura 2

\section{Componentes del ecosistema empresarial visto desde los recursos}

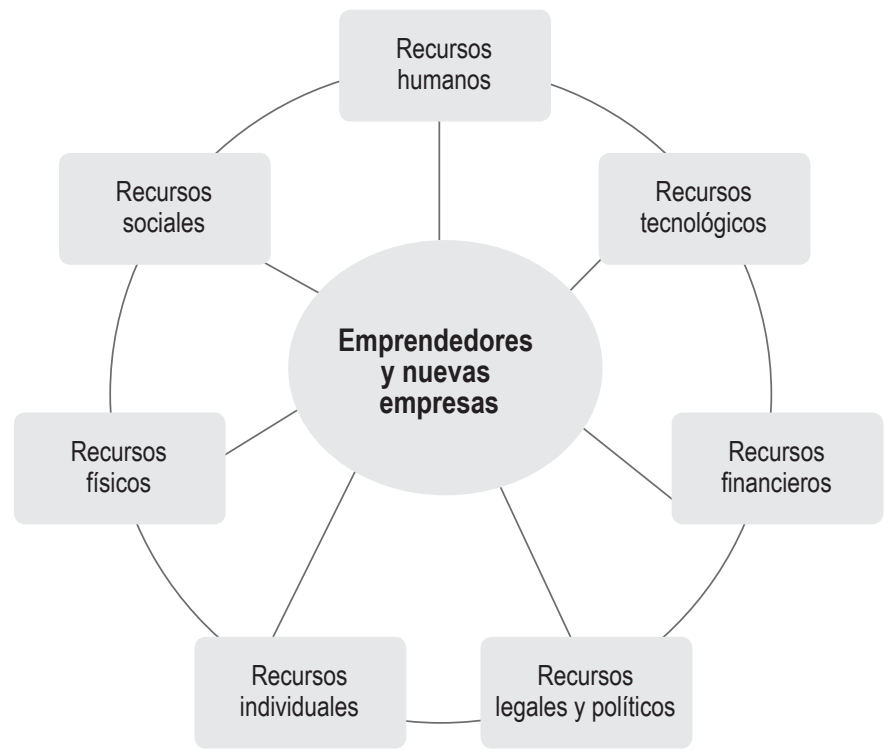

Fuente: García y García (2010).

Elaboración propia.

personales como pueden ser la disciplina y la constancia para estudiar. Un ecosistema empresarial funcionará mejor si las personas que lo componen se preparan, si la preparación es de alto nivel y si los conocimientos adquiridos se ponen a prueba para lograr los mejores resultados.

En el grupo de recursos sociales se le debe dar más relevancia a las redes sociales (Valenzuela 2013). Por un lado las que han sido heredadas porque los familiares antecesores forjaron amistades, interlocutores, conocidos, cuyos contactos hicieron trascender a las generaciones posteriores. También las redes que han sido construidas, pues la debilidad a veces es que se descuida su mantenimiento y se pierden potencialidades de miembros de la red que pueden ser claves para progresar. La pertenencia a instituciones, organizaciones y grupos crea inmersión de hecho, pero como el crecimiento de los agentes 
de intercambio se va haciendo muy grande, es indispensable identificar dónde no se debe producir desgaste y enfocar las energías, el tiempo y la interacción en los puntos de la red que sugieran un buen balance del dar y recibir (Cornejo y Tapia 2011). También las redes que aparecen como consecuencia de la interacción fortuita (el vecino del avión, el compañero del curso de inglés, el proveedor del computador), pues estos agentes tienden a verse como intrascendentes, pero pueden representar puntos de palanca valiosos.

En el grupo de recursos tecnológicos es primordial para los nuevos emprendedores identificar las fuentes de tecnología (Pérez y Dressler 2007), bien para acceder o bien para comprarla. Algunas de estas fuentes son: instituciones públicas y privadas, centros de investigación, incubadoras, universidades, cámaras de comercio e industria, empresas, parques tecnológicos, bibliotecas, centros de experimentación, laboratorios e incubadoras. Como está en juego el conocimiento (Pérez y Dressler 2007), se deben definir claramente en el momento justo y asuntos tales como las condiciones de contratación (casos spin-off), protección de patentes, momento de graduación, tipo de acompañamiento y costos de consecución de la tecnología. Algunas fuentes de tecnología son muy cerradas y operan con baja disposición al uso libre. Los emprendedores deberán buscar las estrategias más pertinentes para llegar a estas fuentes mientras se construye y ejecuta la política pública que rompa con este tipo de asimetrías. De todas maneras, una vez con la tecnología a disposición es imprescindible saberla utilizar y sacarle el mayor provecho posible. Con este propósito, el trabajo en equipo al interior de la empresa naciente será primordial, así como las alianzas con los stakeholders o colindantes.

En el grupo de recursos físicos e intangibles del ecosistema empresarial se debe enfatizar en la construcción o revitalización de hidroeléctricas, vías, puertos, zonas francas, acometidas energéticas y ductos para fluidos hídricos, pues su ausencia o debilidad inhibe la aparición de empresas y también trunca la perennidad de las que nacen (Kelley, Singer y Herrington 2016). En este grupo de recursos inciden varios actores institucionales, pero el más visible y responsable es, sin duda, el gobierno, por lo que se hace indispensable hacer una labor profunda y sistemática desde la adolescencia sobre males como la corrupción, la administración deficiente, el desperdicio del tiempo, la ausencia de procesos rigurosos de selección de personal para el trabajo en el sector 
público, y otros similares. Sin embargo, las empresas privadas, desde las agremiaciones, deben exigir la utilización honesta y bien orientada de los dineros públicos y hacer las veces de ente de control para supervisar que las obras se hagan con diligencia y calidad. En paralelo, debe trabajarse en la existencia y aprovechamiento de recursos intangibles valiosos y necesarios en el ecosistema emprendedor; algunos son el conocimiento, una buena gestión, buenos hábitos de vida de la comunidad, buena seguridad social, atención de calidad en salud, cultura de progreso y solidaridad (Crum y Chen 2015).

En el grupo de recursos financieros, además de los bancos, capitales de riesgo, inversores ángeles, fondos de capital de riesgo y fondos de desarrollo regional, se debe procurar la estructuración y apropiado funcionamiento de los fondos familiares, los fondos de empleados y los ahorros personales. Para esto es esencial que los emprendedores puedan acceder a la formación que los oriente profesionalmente en la consolidación y utilización de estos recursos (Matiz y Fracica 2011). En este grupo el sistema financiero ha tenido debilidades en lo que compete a: trámites exagerados, intereses desestimulantes, competencia exagerada entre agentes que son fuentes de recursos financieros, proliferación de información engañosa y ausencia de compensaciones por cambios favorables a las fuentes. Todas estas situaciones se mantendrán si los usuarios no manifiestan su descontento ante ellas y si la política de gobierno es permisible con estas conductas. La línea del sistema financiero orientada a apoyar el surgimiento de nuevas empresas requiere una legislación que favorezca los emprendimientos con innovación y potencial de crecimiento, y cuyos productos o servicios garanticen una función realmente positiva para la comunidad (Trivedi 2010). Adicionalmente, debe considerar la dosificación de las asignaciones de capital a fin de que estos lleguen a diversas personas y proyectos empresariales. Es indispensable el fomento de más capital semilla, más concursos de emprendimiento y otras modalidades de acceso al capital creador. Adicionalmente, se requiere que las entidades que gestionan los recursos financieros estén en contacto con los otros actores del ecosistema empresarial para que su labor sea más objetiva y provechosa.

En el grupo de recursos legales y políticos tienen presencia muchos temas de gran relevancia. En cuanto a los trámites de constitución de empresas, si bien se han producido avances en la reducción del número de diligencias, se requieren mejores explicaciones en algunos de ellos para que los nuevos 
emprendedores los entiendan con claridad y procedan con confianza a realizarlos. El tema de respeto a las marcas se ha alcanzado parcialmente, pero falta mucho por mejorar, pues aún se presentan diversidad de violaciones que no son atendidas por las entidades privadas o públicas a las cuales les compete esta responsabilidad. Hay otros temas que aún son débiles en el ecosistema empresarial y que pertenecen a este grupo: el desconocimiento de los emprendedores sobre la fuerza que tienen los estatutos de la empresa; lo costoso que resulta hacer protocolos familiares ágiles y aplicables a las pequeñas y medianas empresas; el desconocimiento por falta de difusión desde el gobierno, o por desidia de los emprendedores, de las leyes estrechamente relacionadas con el entrepreneurship y con la actividad empresarial en general (Kelley, Singer y Herrington 2016); el desconocimiento de los emprendedores de la estructura del sector en el que opera u operará, sus tendencias, sus fortalezas, su marco legal, sus oportunidades, la competencia, los efectos de la economía; y las falencias del sistema educativo para formar a los individuos y las familias en aspectos básicos pero claves del conocimiento legal que deberían tener.

El grupo de recursos individuales es completamente novedoso y originario de este trabajo, porque compete a la conducta propia del emprendedor para conciliar el propio yo con los otros componentes del ecosistema empresarial y que de verdad los pueda aprovechar. En este grupo destaca la voluntad para emprender (Alonso y Galve 2008), esto es, para dar inicio a la empresa, para acoger las normas, para aprender por su propia cuenta como un autodidacta, para continuar a pesar de los tropiezos, para gestionar bien los recursos sin dejarse llevar por tentaciones, para dejarse cuestionar por quienes le son cercanos. También es de vital importancia que la autoestima sea cultivada, pues es esta la que le permite al emprendedor moverse con confianza en sus propias capacidades, en su conocimiento y en sus habilidades en su propio ser como persona. Adicionalmente, debe tener presencia la propensión al riesgo moderado, como una conducta que le permita afrontar la incertidumbre con esperanza al emprendedor, yendo al alcance de realizaciones de diverso tipo (Shapero 1982). En este grupo también se debe considerar la mirada positiva que los emprendedores tengan sobre el concepto de trabajo, que lo tomen como algo bueno, interesante y agradable. En el mundo de los negocios estas competencias personales son de vital importancia. Los otros componentes del ecosistema empresarial pueden estar presentes, pero sin la disposición individual para asumir positivamente lo que está a su alre- 
dedor y ponerlo a funcionar creativamente, poco se logrará, ocasionando un desperdicio de recursos y potencialidades personales.

Como se ha expuesto con anterioridad, es indispensable que estos siete elementos interactúen entre sí, respaldados fuertemente por unas sanas políticas de gobierno y la consecución de mercados atractivos, amplios y fieles. Siendo esto posible, se sugiere aquí una definición adaptada desde las disertaciones ya abordadas: un ecosistema empresarial es el conjunto de actores, condiciones y requerimientos físicos e intangibles que interactúan intencionalmente para favorecer, con su capacidad y disponibilidad, la conversión de personas en empresarios, así como el surgimiento de empresas nuevas y la continuidad de las que están en sus primeras etapas. Visto de esta manera, se hace necesaria una cultura transversal de desarrollo del entrepreneurship que haga concienciar a las personas sobre ser empresario como una alternativa de autorrealización personal y profesional, y que estas personas, desde el papel institucional que desempeñen, faciliten los recursos disponibles o conseguibles, en alianza con otras instituciones, para posibilitar el surgimiento de nuevas empresas, fuertes desde el principio y con claridad en sus propósitos sociales y de resultados financieros.

\section{Conclusiones y futuras investigaciones}

Las distintas consideraciones expuestas en este trabajo permiten enunciar tres grandes conclusiones. La primera conclusión lleva a reconocer que son sanos los esfuerzos que se están haciendo para aprender sobre lo que debe ser un ecosistema emprendedor y cómo debe ser el funcionamiento apropiado para que se generen resultados útiles para los emprendedores y para la sociedad. Las bondades radican en que el ecosistema emprendedor conduce a la integración e interacción ordenada de actores, recursos, acciones, entorno, conocimiento, acuerdos, instrumentos y voluntades, complementándose y logrando más que si se actúa de manera independiente.

La segunda conclusión es que, aun teniendo un amplio panorama de lo que es un ecosistema empresarial, falta mucho por recorrer. Nada más si se piensa en cual debería ser aquella etapa del proceso empresarial que prioritariamente debe apoyarse, se puede evidenciar una amplia cantera de cono- 
cimiento por desarrollar, pues en el ecosistema empresarial tendrán protagonismo: el emprendedor que desea crear una empresa pero aún no tiene una idea definida, el emprendedor que está evaluando su idea, el emprendedor que recién ha comenzado, el emprendedor que lleva algún tiempo operando pero requiere todavía ajustes en su proyecto empresarial y, adicionalmente, el apoyo del cuál es responsable el ecosistema empresarial. También podría aludirse el tema de subsidios que se otorguen desde las instituciones públicas, con apoyo de política pública, otorgados a actores privados del sistema; algunos querrían recibir más para hacer su labor eficientemente en el ecosistema. La manera de medir esto para que las unidades de gobierno procedan con objetividad requiere investigación.

Una tercera conclusión es que un enfoque desde los recursos puede facilitar el diseño, análisis y comprensión del ecosistema empresarial, tomando la cultura y los mercados como dimensiones transversales de la totalidad de los siete componentes. De este modo, en este trabajo todos los dominios del ecosistema empresarial propuestos por Isenberg (2010) han sido abarcados $\mathrm{y}$, además, complementados. Adicionalmente, en este trabajo se ha sugerido el grupo de recursos individuales desatendido en la literatura. En realidad lo que está al interior del emprendedor, su forma de pensar, sus sentimientos, sus intereses y su voluntad, son fundamentales para que el ecosistema empresarial pueda ser provechoso. Se pueden hacer muchos planteamientos, hacerlos realidad y ponerlos en funcionamiento, pero sin el interés y voluntad del individuo no se logrará la efectividad y eficiencia deseada para el ecosistema empresarial.

Algunos de los temas en los que sería valioso el interés de futuras investigaciones son los siguientes: ¿De qué manera deben conformarse los comités interinstitucionales que deliberen y decidan sobre el funcionamiento operativo y financiero de los ecosistemas empresariales? ¿Cómo se facilitarán los accesos a los diferentes apoyos del ecosistema empresarial para aquellos que teniendo alto potencial personal y en sus proyectos no posea capacidad de pagar inicialmente los servicios? ¿Cuáles diferencias sustanciales son manifiestas entre un ecosistema empresarial corporativo, otro universitario y otro de región/país? ¿De qué manera se comprometerá responsablemente a los actores del ecosistema empresarial para que obren objetivamente y bajo una conducta correcta? ¿Cuáles serán los límites en la reglamentación de un ecosistema empresarial para que la convivencia no sea ni demasiado libre ni 
demasiado inhibidora? ¿Qué tipo de luchas de poder son susceptibles de presentarse entre los actores, en su búsqueda de protagonismo en el ecosistema, y cómo podrían aminorarse? ¿Cuáles deberían ser los lineamientos de un marco de trabajo sobre ecosistemas empresariales? ¿Cuáles alcances debería proponerse la medición de ecosistemas empresariales? ¿Cómo debería operar el gobierno de un ecosistema empresarial para hacerlo más objetivo, justo y eficiente en sus decisiones, acciones y resultados?

\section{Referencias}

Acs, Zoltan, Lásló Szerb y Erkko Autio. 2015. "National Systems of Entrepreneurship". SpringerBriefs in Economics, No. 50: 13-26.

Alonso, María Jesús, y Carmen Galve. 2008. "El emprendedor y la empresa: una revisión teórica de los determinantes a su constitución". Acciones e Investigaciones Sociales, No. 26: 5-44.

Audretsch, David, y Roy Thurik. 2004. "A Model of the Entrepreneurial Economy". International Journal of Entrepreneurship Education, No. 2: 143-166.

Auletta, Nunzia, y Clariandys Rivera. 2011. "Un ecosistema para emprender". Debates IESA, No. 16: 12-17.

Autio, Erkko, y otros. 2014. "Entrepreneurial Innovation: The Importance of Context". Research Policy 43, No. 7: 1097-1108.

Čalopa, Klačmer, Jelena Horvat y Maja Lalic. 2014. “Analysis of Financing Sources for Startup Companies”. Management 19, No. 2: 19-44.

Cantwell, John, y Katherina Glac. 2004. "La estrategia tecnológica de las empresas multinacionales y el desarrollo de capacidades tecnológicas locales". Cuadernos de Economía y Dirección de la Empresa, No. 20: 83-102.

Capelleras, Joan, y Daniel Kantis, edit. 2009. Nuevas empresas en América Latina: factores que favorecen su rápido crecimiento. Barcelona: Universidad Autónoma de Barcelona / Universidad General Sarmiento.

Carvalho, Luisa, Teresa Costa y Pedro Dominguinhos. 2010. "Creating an Entrepreneurship Ecosystem in Higher Education". En Safeeullah Soomro, edit., New Achievements in Technology Education and Development: 1-16. Consulta: abril de 2016. 〈http://cdn. intechweb.org/pdfs/10542.pdf).

Clarysse, Bart, y otros. 2014. "Creating Value in Ecosystems: Crossing the Chasm Between Knowledge and Business Ecosystems". ERC Research Policy 43, No. 7: 1097-1274.

Cornejo, Marqueza, y María Lourdes Tapia. 2011. "Redes sociales y relaciones interpersonales en internet". Fundamentos en Humanidades, No. 12: 219-229. 
Crum, Michael, y Yi Chen. 2015. "Self-Employment and Subjective Well-being: A Multi-country Analysis". International Journal of Entrepreneurship, No. 19: 15-28.

Diaconu, Mihaela, y Amalia Dutu. 2015. "The Role of the Modern University in Supporting the Entrepreneurial Ecosystem”. European Journal of Interdisciplinary Studies, No. 7: 11- 24.

Duque, Yenni, Marleny Cardona y Jaime Rendón. 2013. "Responsabilidad social empresarial: teorías, índices, estándares y certificaciones”. Cuadernos de Administración, No. 29: 196-206.

Duricova, Grünhagen, y otros. 2014. "University-based Entrepreneurial Ecosystems: Regional Specifics in Eastern and Western Europe". Documento de trabajo. 5th Central European Conference in Regional Science. Košice: Consulta: mayo de 2016. 〈http://www3.ekf. tuke.sk/cers/files/zbornik2014/PDF/Duricova\%20Grunhagen\%20Bischoff.pdf〉.

García, Antonia, y María Gracia García. 2010. "Ecosistema emprendedor para las empresas de base tecnológica: visión basada en los recursos". Tec Empresarial 4, No. 1: 8-21.

Graham, Ruth. 2014. Creating University-Based Entrepreneurial Ecosystems: Evidence from Emerging World Leaders. Massachusetts: MIT Skoltech Initiative.

Hielema, Leslie. 2013. Connecting Entrepreneurs to Success: Optimizing an Entrepreneurial Ecosystem. Orlando: Regional Chamber of Commerce.

Isenberg, Daniel. 2010. "How to Start an Entrepreneurial Revolution". Harvard Business Review, No. 6: 40-51.

---. 2013. Worthless, Impossible and Stupid: How Contrarian Entrepreneurs Create and Capture Extraordinary Value. Boston: Harvard Business Review Press.

Kantis, Hugo, Juan Federico y Sabrina Ibarra. 2014. Índice de condiciones sistemáticas para el emprendimiento dinámico: una herramienta para la acción en América Latina. Buenos Aires: Prodem.

Kelley, Donna, Slavica Singer y Mike Herrington. 2016. 2015 Global Report: Global Entrepreneurship Monitor Association. Consulta: abril de 2016. 〈https://issuu.com/gemcarib bean/docs/gem_global_report_2014〉.

Leslie, Stuart. 2000. "The Biggest Angel of Them All: The Military and the Making of Silicon Valley". En Martin Kenney, edit., Understanding Silicon Valley: The Anatomy of an Entrepreneurial Region: 48-70. Palo Alto: Stanford University Press.

Mason, Colin, y Ross Brown. 2014. "Entrepreneurial Ecosystems and Growth Oriented Entrepreneurship”. Documento de trabajo. Ministerio Holandés de Asuntos Económicos, La Haya. Consulta: abril de 2016. 〈http://www.oecd.org/cfe/leed/entrepreneurial-ecosystems.pdf〉.

Matiz, Francisco Javier, y German Fracica. 2011. "La financiación de nuevas empresas en Colombia: una mirada desde la demanda". Escuela de Administración de Negocios, No. 70: 118-131.

Matthews, Judy. 2002. "Cluster of Innovative Firms: Absorptive Capacity in Larger Networks?". Documento de trabajo. Australian and New Zealand Academy of Management-La Trobe University, Victoria. Consulta: marzo de 2016. «https:/eprints.qut.edu.au/ 14633/1/14633.pdf〉.

Mazzarol, Tim. 2014. "Growing and Sustaining Entrepreneurial Ecosystems: What They Are 
and the Role of Government Policy". Documento de trabajo. Small Enterprise Association of Australia and New Zealand, Fremantle. Consulta: marzo de 2016. 〈http://seaanz.org/ sites/seaanz/documents/reports/SEAANZ_WP_01_2014_Mazzarol.pdf〉.

Monteanu, Sebastian, Irina Iamandi y Eugeniu Tudor. 2015. "Evaluation Model of the Entrepreneurial Character in EU countries". Amfiteatru Economic 17, No. 38: 76-92.

Moore, James. E. 1993. "Predators and Prey: A New Ecology of Competition". Harvard Business Review, No. 71: 73-86.

Nambisan, Satish, y Robert Baron. 2013. "Entrepreneurship in Innovation Ecosystems: Entrepreneurs Self-regulatory Processes and their Implications for New Venture Success". Entrepreneurship Theory \& Practice 37, No. 5: 1071-1097.

Ortmans, Jonathan. 2015. Global Entrepreneurship Network 2015: Impact Report. Consulta: marzo de 2016. 〈https://www.gruenderwoche.de/fileadmin/gew/downloads/ueber-gruen derwoche/global-entrepreneurship-network-impact-report-2015.pdf〉.

Osorio, Fabián, y Fernando Pereira. 2011. "Hacia un modelo de educación para el emprendimiento: una mirada desde la teoría social cognitiva". Cuadernos de Administración 24, No. 43: 13-33.

Parker, Simon, y David Storey. 2010. "What Happens to Gazelles?: The Importance of Dynamic Management Strategy". Journal of Small Business Economics 35, No. 2: 203-226.

Pérez, Daniel, y Matthias Dressler. 2007. "Tecnologías de la información para la gestión del conocimiento". Intangible Capital 15, No. 3: 31-59.

Porter, Michael. 1998. Cluster and the New Economics of Competition. Boston: Harvard Business Review.

Shapero, Albert, y Lisa Sokol. 1982. "Some Social Dimensions of Entrepreneurship". En Calvin Kent, edit., Encyclopedia of Entrepreneuship: 72-90. Nueva Jersey: Prentice Hall.

Teece, David, y otros. 1994. "Understanding Corporate Coherence: Theory and Evidence". Journal of Economic Behavior and Organization 23, No. 1: 1-30.

Trivedi, Chitvan. 2010. "Towards a Social Economical Framework for Social Entrepreneurship". The Journal of Entrepreneurship 19, No. 1: 63-80.

Valdés, Esperanza, y Rosa María Vidal, coord. 2012. Guía del ecosistema del emprendedor. Madrid: Centro de Iniciativas Emprendedoras de la Universidad Autónoma de Madrid.

Valenzuela, Rebeca. 2013. "Las redes sociales y su aplicación en la educación”. Revista Digital Universitaria 14, No. 4: 1-14.

Venkataraman, Sankaran. 2004. "Regional Transformation Through Technological Entrepreneurship". Journal of Business Venturing 19, No. 1: 153-167.

Willer, Claire, y Andy Neely. 2013. "Business Model Design in an Ecosystem Context". Documento de trabajo. University of Cambrigde, Cambrigde. Consulta: marzo de 2016. «https:/ /cambridgeservicealliance.eng.cam.ac.uk/resources/Downloads/Monthly\%20Papers/ 2013JunepaperBusinessModelDesigninEcosystemContext.pdf〉.

Zott, Cristoph, y Raphael Amit. 2010. "Business Model Design: An Activity System Perspective”. Long Range Planning, No. 43: 216-226. 